\title{
Pneumomediastinum As A New Rare Complicance of Covid-19 Pneumonia: CT Findings
}

\author{
Francesco Messina* \\ Clinical Applications of Cardiovascular Imaging, MD at Radiology Unit of Riuniti Hospital, Italy
}

*Corresponding author: Francesco Messina, Radiology Unit - Riuniti Hospital, Azienda Ospedaliera Grande Ospedale Metropolitano "Bianchi-Melacrino-Morelli", Reggio Calabria, Italy.

To Cite This Article: Francesco Messina, Pneumomediastinum As A New Rare Complicance Of Covid-19 Pneumonia: CT Findings. Am J Biomed Sci \& Res. 2021 - 12(3). AJBSR.MS.ID.001758. DOI: 10.34297/AJBSR.2021.12.001758.

Received: 眥 March 16, 2021; Published: 軘 April 06, 2021

\begin{abstract}
The Covid-19 Coronavirus infectious pandemic began since December 2019, mainly causing bilateral interstitial pneumonia in affected patients. The heterogeneity of the course and clinical frameworks of the pathology represents an important problem for health providers, who are faced with both the acute phase of the disease and the possible complications. An optimal management of patients was, initially, very hard. So, the use of computed tomography (CT) imaging in the diagnosis and follow-up had rapidly grown, and radiological patterns along the disease course were increasingly understood. Many typical imaging features of this disease were described: bilateral multilobar ground-glass opacity (GGO), with a prevalent peripheral or posterior distribution, mainly in the lower lobes; consolidative areas of the lungs; septal thickenings; bronchiectasis; pleural thickenings and subpleural involvements. Unenhanced chest CT is considered the first-line imaging tool for the diagnosis, the severity assessment, and monitoring of Covid-19 pneumonia [1]. Spontaneous pneumomediastinum (SPM) is a rare complication of Covid-19 pneumomia. The major risk factors for development of SPM are male gender, advanced age and preexisting lung disease. SPM was considered as a potential predictive factor for adverse outcome and probable cause of unexplained deterioration of clinical conditions in Covid-19 pneumonia [2,3] (Figure 1).
\end{abstract}

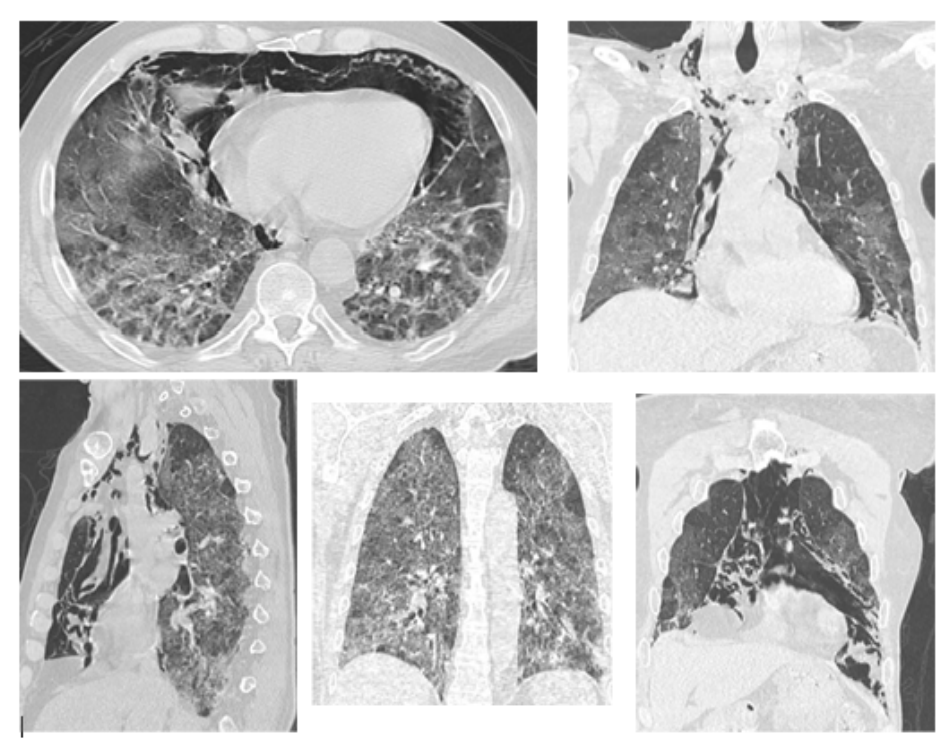

Figure 1: CT of a 70-year-old male patient showed, bilaterally, multiple and diffuse thickenings with a "ground glass" (GGO) pattern, and bilateral pulmonary interstitial-septal reticular thickenings, with bi-basal sub-pleural consolidations and fibrotic streaks. CT also showed a new, important, finding: a severe pneumomediastinum in all mediastinal compartments, most evident anteriorly, with evidence of concomitant soft tissue emphysema extending to the basal regions of the neck, bilaterally. 


\section{Discussion}

The clinical course of the Covid-19 infection is unpredictable and varies from asymptomatic or subclinical symptoms to severe disease, with development of acute respiratory failure and organ failure. Due to high sensitivity and rapid access, chest CT had an important role in diagnosis and management of Covid-19 infection. The causes of pneumomediastinum may also be related to the abrupt increase in intrathoracic pressure associated with Valsalva maneuver (cough, vomiting, vigorous activity) and subsequently rupture of the alveoli, followed by air dissection through the broncho vascular bundles into the mediastinum (Macklin's effect) [4]. A possible explanation of the pneumomediastinum could be linked to a particular elasticity / fragility of the lung adjacent to the mediastinum, which, stressed by high pressure values induced by persistent coughing, tends to cause ruptures of preexisting subpleural "blebs". Another hypothesis could be that the anterior pulmonary areas are those most stressed by the pressure increases induced by persistent cough, and all this, together with the syncytial metaplasia induced by Covid-19 disease, caused a pneumomediastinum [5]. Another important hypothesis to explain the pneumomediastinum can be traced back to the "lung entrapment" theory, which generates an increase in the elastic recoil pressures of the lung. The "lung entrapment" theory is related to the pathological situations in which the lung becomes rigid and unhelastic (such as broncho-obstruction athelettasy; Covid-19 disease), resulting in complete pulmonary parenchymal subversion and the onset of pneumomediastinum. Therefore, grouping together the concepts expressed above, I believe that there are three possible physio pathological explanations for the onset of pneumomediastinum:

I. "lung entrapment" theory, which includes an interstitial commitment, with a rigid and unhelastic lung.

II. "heavy" fan maneuvers (such as high flows).

III. embolic vascular defect: in case of Covid-19 bilateral pneumonia, the pathological mechanisms of the pulmonary vascular district (thrombotic and hemorrhages) can increase the weight of the lungs, resulting in a reduction in the relative elastic lung capacity (reduction of compliance). This condition, together with the interstitial inflammatory mechanisms, could result in a greater weight load on some pleural districts (based on the extent and localization of disease), and if this mechanism with respiratory acts exceeds the resistance capacity of the negative pressure in force between the pleural leaflets, the pleura can yield, determining (even with the complicity and the additional stress of ventilation manoeuvres even at "soft" pressures) a find of pneumomediastinum that feeds on its own over time.

\section{Conclusion}

Computed Tomography is important not only in the early stages of Covid-19 diagnosis, but also in the evaluation of complications and concomitant pathologies during the subsequent course of the disease, orienting toward the best clinical management/outcome for the patient.

\section{Acknowledgement}

None

\section{Conflicts of Interest}

No conflicts of interest

\section{References}

1. (2020) ACR recommendations for the use of chest radiography and CT for suspected Covid-19 infection.

2. Brogna B, Bignardi E, Salvatore P, Alberigo M, Brogna C, et al. (2020) Unusual presentations of Covid19 pneumonia on CT scans with spontaneous pneumomediastinum and loculated pneumothorax: a report of two cases and a review of the literature. Heart Lung 49(6): 864-868.

3. López Vega JM, Parra Gordo ML, Diez Tascón A, Ossaba Vélez S (2020) Pneumomediastinum and spontaneous pneumothorax as an extrapulmonary complication of Covid-19 disease. Emerg Radiol 27(6): 727-730.

4. Macklin CC (1939) Transport of air along sheaths of pulmonic blood vessels from alveoli to mediastinum. Arch Intern Med 64: 913.

5. Ackermann M, Verleden SE, Kuehne M, Haverich A, Welte T, et al. (2020) Pulmonary vascular endothelialitis, thrombosis, and angiogenesis in Covid-19. N Engl J Med 383: 120-128. 